\title{
A Joint Doppler Frequency Shift and DOA Estimation Algorithm Based on Sparse Representations for Colocated TDM-MIMO Radar
}

\author{
Tao Chen, Huanxin Wu, and Lutao Liu \\ College of Information and Communication Engineering, Harbin Engineering University, Harbin 150001, China \\ Correspondence should be addressed to Lutao Liu; liulutao@msn.com
}

Received 26 July 2014; Accepted 29 September 2014; Published 16 November 2014

Academic Editor: Zhiguang Feng

Copyright (C) 2014 Tao Chen et al. This is an open access article distributed under the Creative Commons Attribution License, which permits unrestricted use, distribution, and reproduction in any medium, provided the original work is properly cited.

\begin{abstract}
We address the problem of a new joint Doppler frequency shift (DFS) and direction of arrival (DOA) estimation for colocated TDM-MIMO radar that is a novel technology applied to autocruise and safety driving system in recent years. The signal model of colocated TDM-MIMO radar with few transmitter or receiver channels is depicted and "time varying steering vector" model is proved. Inspired by sparse representations theory, we present a new processing scheme for joint DFS and DOA estimation based on the new input signal model of colocated TDM-MIMO radar. An ultracomplete redundancy dictionary for angle-frequency space is founded in order to complete sparse representations of the input signal. The SVD-SR algorithm which stands for joint estimation based on sparse representations using SVD decomposition with OMP algorithm and the improved M-FOCUSS algorithm which combines the classical M-FOCUSS with joint sparse recovery spectrum are applied to the new signal model's calculation to solve the multiple measurement vectors (MMV) problem. The improved M-FOCUSS algorithm can work more robust than SVD-SR and JS-SR algorithms in the aspects of coherent signals resolution and estimation accuracy. Finally, simulation experiments have shown that the proposed algorithms and schemes are feasible and can be further applied to practical application.
\end{abstract}

\section{Introduction}

It is a novel technology and worth studying deeply that multiple-input multiple-output (MIMO) radar architecture is applied to autocruise and safety driving system $[1,2]$. Using this technology we hope distance, velocity, and direction of arrival (DOA) of multisource signals reflected by other automobiles near your own car can be measured simultaneously. According to these estimated parameters the auto can take measures to deal with all kinds of situations automatically or warn the driver. MIMO radar architecture with colocated transmitter (TX) and receiver (RX) antennas has a larger virtual aperture [3]. The basic idea of virtual aperture is shown in Figure 1. Here, we consider a uniform linear array (ULA) with $M=4$ transmitters, $N=4$ receiver antennas, and the receiver array spacing $d$. According to MIMO theory we know that its virtual array consists of 16 virtual antennas in Figure 1. So it can achieve a more accurate DOA and improve multisource resolution in the same time. In recent years sparse representations and compressed sensing algorithm have been widely studied and some algorithms based on sparse representations have been applied to DOA estimation for MIMO radar [3-7]. DOA estimation algorithm based on sparse representations and reconstruction theory has two important advantages. One is that it can process coherent signal; the other is that it can complete DOA estimation in few snapshots.

Generally speaking, MIMO radars require more complex hardware [8] when they can be realized by code, frequency. MIMO system requires that the number of receiver channels should be equal to the number of receiver antennas. Moreover, a difference in gain and phase between the receiver channels deteriorates the accuracy significantly [8]. Meanwhile customers also demand an increased performance of automotive radar sensor at low sensor costs with the development of SiGe MMIC technology $[2,9]$. The architecture of the time division modulated (TDM) for MIMO (TDMMIMO) radars may be utilized for the purpose of economic 


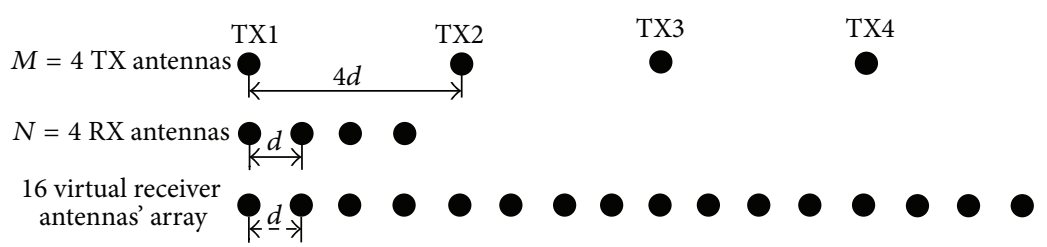

FIGURE 1: MIMO radar system with $M=4$ transmitters and $N=4$ receiver antennas and its virtual array consists of 16 antennas.

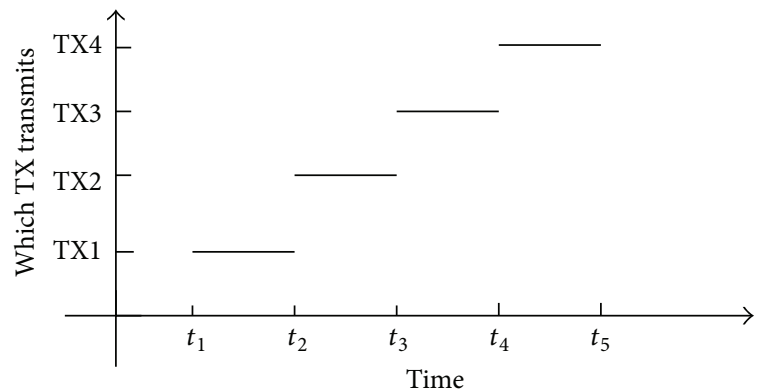

FIgURE 2: Example of a TDM scheme: 4 transmitters transmitting at times. Here $\underline{t}=\left[t_{1}, t_{2}, t_{3}, t_{4}\right]^{T} ; \underline{d}^{\text {Pulse }}=\left[d_{1}^{\mathrm{TX}}, d_{2}^{\mathrm{TX}}, d_{3}^{\mathrm{TX}}, d_{4}^{\mathrm{TX}}\right]^{T}$.

and simple system design for autocruise and safety driving system. Some receiver or transmitter channels may be cut only by depending on switching different RX or TX antennas in time domain.

A MIMO radar has the ability to transmit different, noncoherent orthogonal signals from different positions using an array of multiple transmitter antennas [10]. If TX antennas could transmit signal at different times, only one TX antenna is permitted to transmit signal during each period. This MIMO radar system can be called as TDM-MIMO radar system. For example, $4 \mathrm{TX}$ antennas may transmit signal at the schedule in Figure 2. Note that the signal phase related to other receiver channels can be varying even if we are facing one incident signal just because TX antennas transmit signal at different time instants in one cycle and the target moving conduces Doppler frequency shift. The steering vector model of colocated TDM-MIMO radar is "time varying steering vector" model [8]. Hence, the conventional algorithms such as Capon and MUSIC [11-13] cannot process this kind of input signal model directly. It is also very difficult to precisely estimate DFS in advance in a short time for the practical application of TDM-MIMO radar.

In recent years a new highly integrated SiGe MIMO radar sensor has been developed [2, 14-16]. In these systems the frequency-modulated continuous wave (FMCW) will be radiated by transmitters. So DFS and high resolution DOA estimation technology research are very important requirements for the application in autocruise and safety driving system $[16,17]$. The specification of the automotive long range radar (LRR) sensor includes a high DOA estimation accuracy of $\theta_{\text {err }} \leq 0.4^{\circ}$, an angular resolution of $\Delta \theta \leq 3^{\circ}$, and target ranges $R$ up to $200 \mathrm{~m}$ with relative target velocities $v$ between $-60 \mathrm{~m} / \mathrm{s}$ and $20 \mathrm{~m} / \mathrm{s}$ [18].
Facing the aforementioned applications and problems of parameters estimation for TDM-MIMO radar, some researchers proposed some methods to solve these problems. For time-invariant steering vector model, the conventional algorithms such as Capon beamformer [8], AM [18], and MUSIC [16, 18] were applied. For time varying steering vector model, the Cramer-Rao bound (CRB) of parameter estimation for a TDM-MIMO radar with colocated antennas was computed and an optimal TDM scheme was derived $[1,19]$. ISI-SAGE algorithm was tested to estimate DFS and DOA jointly [20]. A new nonstationary version of Capon beamforming was developed for the time varying steering vector model [8].

In this paper, we offer a new insight into the joint DFS and DOA estimation based on sparse representations for time varying steering vector model of colocated TDM-MIMO radar. An ultracomplete redundancy dictionary for anglefrequency space is founded in order to complete sparse representations for the input signal. The SVD-SR algorithm which stands for joint estimation based on sparse representations using SVD decomposition with OMP algorithm and the improved M-FOCUSS algorithm which combines the classical M-FOCUSS algorithm with joint sparse recovery spectrum are applied to the new model to solve MMV problem. By using these algorithms, both the coherent signals and uncorrelated signals can be distinguished successfully and estimation accuracy can be further improved in the case of MMV with low SNR.

This paper is concerned with issues of signal model and signal processing for TDM-MIMO. The remainder of this paper is organized as follows. We present the signal model in Section 2 firstly. Then sparse representations' principle for TDM-MIMO model is discussed and anglefrequency dictionary is designed in Section 3. How to deal with joint DFS and DOA estimation based MMV model using SVD decomposition and improved M-FOCUSS algorithm is demonstrated in detail in Section 4. In Section 5 simulation results and some discussions are given. Finally, we conclude our work in Section 6.

\section{Signal Model}

We consider a colocated MIMO radar consisting of a linear receiver and transmitter array with isotropic antennas. The moving target is modeled as a point source and the transmitted signal is narrowband. The positions of the $N_{\mathrm{RX}} \mathrm{RX}$ antennas and $M_{\mathrm{TX}}$ TX antennas are given in $\underline{d}^{\mathrm{RX}} \in \mathbb{R}^{N_{\mathrm{RX}}}$ and $\underline{d}^{\mathrm{TX}} \in \mathbb{R}^{M_{\mathrm{TX}}}$, respectively, in units of $2 \pi / \lambda$, where $\lambda$ is the 


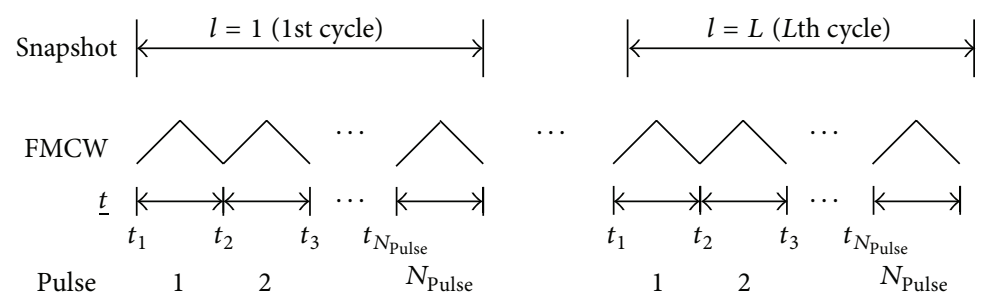

FIgURE 3: Structure of the cycles in data acquisition.

carrier wavelength. The positions of the TX antennas in the sequence in which they transmit are given in $d^{\text {Pulse }} \in \mathbb{R}^{N_{\text {Pulse }}}$. $N_{\text {Pulse }}$ is the number of transmitted pulses in a cycle. The time instance at which the antennas transmit in a cycle is given $\underline{t} \in \mathbb{R}^{N_{\text {Pulse }}}$. This means that the antenna with position $d_{i}^{\text {Pulse }}$ transmits at time $t_{i}$; see Figure 2 for an example. So the steering vector of the virtual array is given by

$$
\underline{a}(u)=\exp \left(j \cdot \underline{d}^{\mathrm{Virt}} u\right)=\exp \left(j \cdot \underline{d}^{\mathrm{Pulse}} u\right) \otimes \exp \left(j \cdot \underline{d}^{\mathrm{RX}} u\right) .
$$

Here $u=\sin (\Theta)$ is the DOA of targets. $y=\exp (\underline{x})$ is understood as an element-by-element operation $y_{i}=$ $\exp \left(x_{i}\right) . \otimes$ is the Kronecker tensor product. - stands for column vector.

We assume a number of $L$ measurement cycles where one cycle consists of $N_{\text {Pulse }}$ pulses. See Figure 3 for an example. $\underline{t}=$ $\left[t_{1}, t_{2}, t_{3}, \ldots, t_{N_{\text {Pulse }}}\right]^{T}$. So the complex baseband signal $y(\bar{l}) \in$ $\mathbb{C}^{N_{\text {Virt }}}$ of cycle $l$ is given as

$$
\underline{y}(l)=\exp \left(-j \cdot \underline{\gamma} \omega_{d}\right) \odot \underline{a}(u) \frac{1}{\sqrt{N_{\text {Pulse }}}} s(l)+\underline{N}(l) .
$$

Here $\odot$ is the entrywise Hadamard product and $N_{\text {Virt }}=$ $N_{\text {Pulse }} \cdot N_{\mathrm{RX}}$ is the number of virtual antennas. $s(l) \in \mathbb{C}$ is the target echo signal. $N(l)$ is additive complex Gaussian noise with zero mean, spatially and temporally uncorrelated with $E\left(\underline{N}(l) \underline{N}^{H}(m)\right)=\delta_{l \cdot m} \sigma^{2} I$ ( ${ }^{H}$ stands for Hermitian transformation). The factor $1 / \sqrt{N_{\text {Pulse }}}$ adjusts the signal strength of one cycle, consisting of $N_{\text {Pulse }}$ pulses, according to a constant transmitting energy. $\omega_{d}$ stands for Doppler frequency shift. Consider

$$
\underline{\gamma}=\underline{t} \otimes \underline{1}_{N_{\mathrm{RX}}} .
$$

Here $\underline{1}_{K}$ is a vector of length $N_{\mathrm{RX}}$ with all elements equal to 1 . With the definition of a new steering vector,

$$
\underline{b}\left(u, \omega_{d}\right):=\exp \left(-j \cdot \underline{\gamma} \omega_{d}\right) \odot \underline{a}(u) .
$$

the signal model can be written as

$$
\underline{y}(l)=\underline{b}\left(u, \omega_{d}\right) \cdot \frac{1}{\sqrt{N_{\text {Pulse }}}} s(l)+\underline{N}(l),
$$

From formula (4), we will see that steering vector for input model of colocated TDM-MIMO radar is "time varying steering vector" model since there is $t$ item existing. In order to complete the parameters estimation we make the following assumptions.
(1) The far field condition is satisfied. So DOA of one target echo signal is the same for all receiver antennas.

(2) The DOA and the moving velocity of the target will not change during one complete measurement. So both DOA and DFS are constants during $L$ measurement cycles. In other words, the parameters' changes have smaller effect on the accuracy of parameters estimation and these changes can be ignored.

(3) We consider a MIMO radar system with colocated antennas; that is, the direction of departure (DOD) and DOA are the same.

(4) Time of switching different TX and RX antennas or channels is accurate; that is, the estimation error of $t$ vector is ignored.

\section{Sparse Representations for TDM-MIMO Receiver Signal}

The signal sparse representations mean that a signal may be represented in an ultracomplete redundancy dictionary. Then we find the best linear combination of atoms to represent original signal. Inspired by the literature [7], we can establish an ultracomplete redundancy dictionary called angle-frequency space to represent original signal $y(l)$ of formula (5). The following is the angle-frequency dictionary $\Psi$ we designed:

$$
\begin{gathered}
\Psi=\left[\begin{array}{cccccc}
\Phi_{1} & \Phi_{2} & \cdots & \Phi_{p} & \cdots & \Phi_{P}
\end{array}\right] \in \mathbb{C}^{N_{\mathrm{Virt}} \times P Q}, \\
\Phi_{p}=\left[\begin{array}{llllll}
\underline{\phi}_{p 1} & \underline{\phi}_{p 2} & \cdots & \underline{\phi}_{p q} & \cdots & \underline{\phi}_{p \mathrm{Q}}
\end{array}\right], \\
\underline{\phi}_{p q}=\exp \left(-j \cdot \underline{\gamma}_{q}\right) \odot \underline{a}(u) .
\end{gathered}
$$

According to formula (6) we can rewrite the signal model of formula (5) to group $L$ snapshots as

$$
Y=\Psi \cdot Z+N,
$$

where $Y=[y(1), y(2), \ldots, y(L)]$ is a matrix of size $N_{\text {Virt }} \times L$, $Z=[z(1), z \overline{(2}), \ldots, z(L)]$ is a matrix of size $P Q \times L$, and $N$ is a noise matrix of size $N_{\text {Virt }} \times L$. Let $\underline{\phi}_{1}, \underline{\phi}_{2}, \ldots, \underline{\phi}_{p q}$ be a set of joint angle-frequency spaces of DFS and DOA space. Here $P Q$ is the total number of vectors of joint angle-frequency space matrix that the algorithm can scan. Assuming that $K$ incident signals exist in the noise-free case, we can find $K$ nonzero coefficients $z_{\theta}(t)=s_{k}(t)$ at positions corresponding 
to the atom $\theta=\theta_{k},\left(k=1,2, \ldots, K, \theta_{k}=p q, p=\right.$ index $\left(\operatorname{DOA}\left(s_{k}\right)\right), q=$ index $\left.\left(\operatorname{DFS}\left(s_{k}\right)\right)\right)$ and zero coefficients at the remaining $P Q-K$; that is, the sparsity in the anglefrequency space denotes that only a few atoms from the dictionary will be required to match the measurements. Here $K \ll P Q$ is needed to be satisfied.

The number of nonzero rows of $Z$ matrix is referred to as the sparsity of the solution. There are many recovery algorithms to reconstruct original signal. Popular approaches include greedy sequential search techniques such as matching pursuit and methods based on minimizing diversity measures such as basis pursuit and FOCUSS [21]. Firstly we use orthogonal matching pursuit (OMP) algorithm [22] described as follows.

Input. The observation vector $y \in \mathbb{C}^{n}$ and a measurement matrix $\Psi=\left\{\underline{\phi}_{i}, i=1,2, \ldots, m\right\} \in \mathbb{C}^{n \times m}$.

Initialization. Index $I=\emptyset$, residual $\underline{r}=y$, and sparse representation $\underline{\widehat{z}}=\underline{0} \in \mathbb{C}^{m}$.

Iteration.

While (stopping criterion false)

$$
\begin{aligned}
& i=\arg \max \left|\left\langle\underline{r}, \underline{\phi}_{j}\right\rangle\right| ; \\
& I=I \cup\{i\} ; \\
& \underline{r}=\underline{y}-[\Psi(:, I)][\Psi(:, I)]^{\dagger} \underline{y} .
\end{aligned}
$$

End while

$$
\widehat{\underline{z}}(I)=[\Psi(:, I)]^{\dagger} y .
$$

Output. Sparse representations $\underline{\widehat{z}}$ and the original signal $y=$ $\Psi \widehat{z}$.

Here $[\bullet]^{\dagger}$ is pseudoinverse matrix transformation. Stopping criterion is that iteration times are equal to the known number $K$ of incident sources (i.e., sparsity). In the TDMMIMO estimation model, we can calculate the DOA and $\omega_{d}$ according to $I$ vector. From $I$ vector we can determine which atom or atoms will be represented; then $p$ and $q$ may be ascertained. From $p$ value DOA may be estimated and $\omega_{d}$ may be estimated from $q$ value successfully.

When TDM-MIMO estimation model has $L$ cycles, that is, $Y=[y(1), \underline{y}(2), \ldots, \underline{y}(L)]$, we can solve $Z$ efficiently using joint sparse recovery algorithms [23]. The joint sparse recovery spectrum of the DOA and DFS can be calculated by

$$
P_{y}(\theta)=\frac{1}{L} \sum_{l=1}^{L}\left\|\widehat{z}_{\theta}(l)\right\|^{2}, \quad \theta=\theta_{1}, \theta_{2}, \ldots, \theta_{P Q} .
$$

So if there are multiple snapshots of the received array signals, we will use joint recovery algorithms to estimate the spectrum as formula $(8)[24,25]$.

\section{Signal Reconstructions Algorithm for MMV Problem}

Only one snapshot $(L=1)$ is needed to estimate parameters in sparse representations theory, but in actual application more snapshots are sampled in order to improve SNR and estimation accuracy. In formula (8) computation burden can become larger and larger with the increase of snapshots and the estimation results can be influenced more easily by SNR of input signals. This problem is so called MMV problem. In order to reduce computational load and improve estimation precision, there are two classical kinds of methods to solve MMV problem. One is dependent on SVD algorithm such as $\ell_{1}$-SVD $[26,27]$; the other is dependent on M-FOCUSS approach. These two algorithms both take datum correlation of different snapshots into consideration.

4.1. Signal Reconstruction Based on SVD. We investigate SVD subspace decomposition method [28, 29] firstly. The following formula is SVD subspace decomposition for $Y$ matrix:

$$
Y=U \Lambda V^{H}=\left[\begin{array}{ll}
U_{s} & U_{N}
\end{array}\right] \Lambda V^{H} .
$$

Size of matrices $U, \Lambda$, and $V$ is $U \in \mathbb{C}^{N_{\text {Virt }} \times N_{\text {Virt }}}, \Lambda \in \mathbb{C}^{N_{\text {Virt }} \times L}$, and $V \in \mathbb{C}^{L \times L} . U_{s} \in \mathbb{C}^{N_{\text {Virt }} \times r}$ is signal subspace corresponding to the larger singular values (assuming $r$ ) in $\Lambda$ matrix. $U_{N} \in$ $\mathbb{C}^{N_{\text {Virt }} \times\left(N_{\text {Virt }}-r\right)}$ is noise subspace corresponding to the smaller singular values (assuming $N_{\text {Virt }}-r$ ) in $\Lambda$ matrix. So we can define

$$
Y_{S}=Y V D_{r}
$$

Here $D_{r}=\left[\begin{array}{lll}\Lambda_{r \times r} & O_{r \times(L-r)}\end{array}\right]^{H}$ is a matrix composed by the larger singular values. Also we define $Z_{S}=Z V D_{r}, N_{S}=$ $N V D_{r}$. So the formula (7) can be espressed as

$$
Y_{S}=\Psi \cdot Z_{S}+N_{S}
$$

Size of matrices $Y_{S}, Z_{S}$, and $N_{S}$ is $Y_{S} \in \mathbb{C}^{N_{\text {Virt }} \times r}, Z_{S} \in \mathbb{C}^{P Q \times r}$, and $N_{S} \in \mathbb{C}^{N_{\text {Virt }} \times r}$. $Y_{S}$ and $Y$ have the same sparsity level. By using SVD algorithm the dimension of observation matrix has been reduced from $N_{\text {Virt }} \times L$ to $N_{\text {Virt }} \times r$. In general $r \ll L$ and $r<N_{\text {Virt }}$ is conditioned. Meanwhile we will achieve good performance of parameters estimation in lower SNR. $\ell_{1}$-SVD algorithm is a successful DOA estimation method for sparse solution. But convex optimization problem such as a second-order cone (SOC) programming has to be utilized in $\ell_{1}$-SVD algorithm, so its computational complexity will increase. Here, we will continue to use OMP greedy algorithm after SVD is done. We generalize the scheme of algorithm based on SVD decomposition with OMP.

Input. The observation matrix $Y$ and a sparse representations matrix $\Psi=\left\{\underline{\phi}_{i}, i=1,2, \ldots, P Q\right\} \in \mathbb{C}^{N_{\text {Virt }} \times P Q}$. Consider the following:

(1) SVD decomposition for matrix $Y$;

(2) determining the number of the larger singular values $(r)$

(3) calculating $Y_{S}=Y V D_{r}$;

(4) using OMP algorithm for each column of matrix $Y_{S}$;

(5) searching the index atoms in atomic dictionary.

Output. DOA and DFS. 
4.2. Signal Reconstruction Based on M-FOCUSS. M-FOCUSS algorithm is developed based on the focal underdetermined system solver (FOCUSS) algorithm [30] developed for the single measurement case and it has an ability to utilize multiple measurement vectors to accurately identify the sparsity structure [21]. According to formula (7), we can summarize the scheme as follows so as to compute $Z$ sparse solution.

Input. The observation matrix $Y$ and a measurement matrix $\Psi=\left\{\phi_{i}, i=1,2, \ldots, m\right\} \in \mathbb{C}^{n \times m}$

Initialization. $X_{1}=1^{m \times L}, \mathcal{\varepsilon}, p$, iternum $=0$.

Iteration.

While $\left(\left\|X_{k+1}-X_{k}\right\|_{2} /\left\|X_{k}\right\|_{2}>\varepsilon\right.$, iternum < $\max$ _iteration)

$W_{k+1}=\operatorname{diag}\left(c_{k}[i]^{1-p / 2}\right)$, with respect to $c_{k}[i]=$ $\left\|X_{k}[i]\right\|_{2}$

$B_{k+1}=\left[A_{k+1}\right]^{\dagger} Y$, where $A_{k+1}=\Psi W_{k+1}$;

$X_{k+1}=W_{k+1} B_{k+1}$;

iternum $=$ iternum +1 .

End while.

Output. Sparse representations matrix $\widehat{Z}=X_{k+1}$.

Here, $\|x\|_{2}=\left(\sum\left|x_{i}\right|^{2}\right)^{1 / 2}$ is Frobenius norm and $\varepsilon$ is threshold to stop the whole algorithm. The parameter $p$ lies in $[0,2]$. According to experiences, the best selection for $p$ lies in $[0.8,1]$ so that convergence rate is good and optimum solution can be achieved easily. The optimal solution is conditioned by the minimum Frobenius norm with weighted values, so matrix $W_{k}$ is the $k$ th iterative weighted matrix.

By using M-FOCUSS algorithm we can achieve sparse representations matrix $\widehat{Z}$ and deal with MMV problem successfully. Furthermore, we can use formula $(8) P_{y}(\theta)=$ $(1 / L) \sum_{l=1}^{L}\left\|\widehat{z}_{\theta}(l)\right\|^{2}$, where $\widehat{z}_{\theta}(l)$ is the row vector of matrix $\widehat{Z}$ to calculate the joint sparse recovery spectrum function. Then the positions of peaks of spectrum function are the DFS and DOA estimation values and number of peaks is the number of input signals with different DFSs and DOAs.

\section{Simulation Experiments}

We present some simulations to verify the theoretical results below. We consider a TDM-MIMO radar with $4 \mathrm{RX}$ and $2 \mathrm{TX}$ antennas, uniformly spaced with an antenna distance of $\lambda / 2$; that is, $\underline{d}^{\mathrm{RX}}=\left[\begin{array}{llll}1 & 2 & 3 & 4\end{array}\right]^{T}$ and $\underline{d}^{\mathrm{TX}}=\left[\begin{array}{ll}0 & 5\end{array}\right]^{T}$. The antennas are laid as shown in Figure 4. The TX antennas transmit signal in TDM schedule and transmit schedule is shown in Figure 5.

We choose 1 cycle with $N_{\text {Pulse }}=4$ pulses and set the transmitting time instants to $\underline{t}=[0,1,2,3]$ in units of ms. So $\underline{d}^{\text {Pulse }}=\left[d_{1}^{\mathrm{TX}}, d_{2}^{\mathrm{TX}}, d_{2}^{\mathrm{TX}}, d_{1}^{\mathrm{TX}}\right]$ will be achieved. The basic simulation parameters are set as follows. The carrier

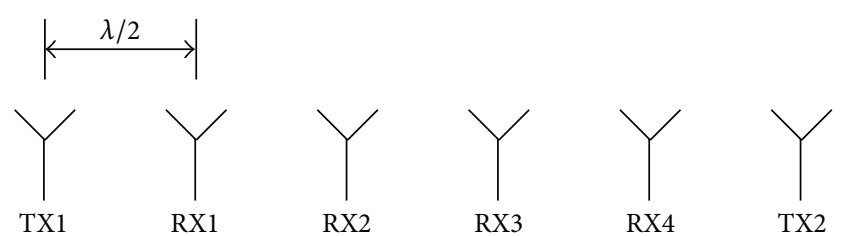

FIGURE 4: Array construction of antennas.

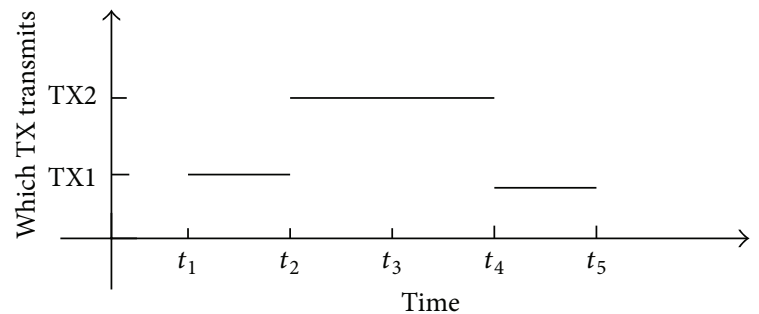

FIGURE 5: TDM scheme: 2 transmitters transmitting at times.

frequency is equal to $77 \mathrm{GHz}$. DOA search range lies in $\left[-60^{\circ}, 60^{\circ}\right]$ with step size $0.1^{\circ}$ and velocity search range lies in $[0 \mathrm{~m} / \mathrm{s}, 100 \mathrm{~m} / \mathrm{s}]$ with step size $1 \mathrm{~m} / \mathrm{s}$. Snapshots are equal to $L=200$. We do two experiments: one is for measurement of the root mean square errors (RMSE) of velocity and DOA estimation in different SNRs; the other is for multisource resolution of the coherent and uncorrelated signals.

5.1. Simulation Experiments of Joint DFS and DOA Estimation RMSE. 500 independent Monte Carlo simulations are carried out for each SNR varying from $0 \mathrm{~dB}$ to $30 \mathrm{~dB}$ with $5 \mathrm{~dB}$ step. The true DOA of incident signal and velocity of target moving are selected randomly in each trial. But DOA and velocity parameters set in one trial are constant. Velocity estimation is shown in Figure 6 and DOA estimation is shown in Figure 7. As we know, the formula $\omega_{d}=2 v / \lambda$, where $v$ is moving velocity of the target. So velocity simulation results are given directly instead of DFS due to the consideration of more concerned velocity parameter in the practical application. JS-RS stands for algorithm of the joint sparse recovery spectrum of DFS and DOA according to formula (8). SVD-RS stands for algorithm of joint estimation based on sparse representations using SVD with OMP algorithm to reconstruction; see formula (11) and scheme. MFOCUSSSR stands for improved M-FOCUSS algorithm with joint sparse recovery spectrum. Root mean square error (RMSE) is defined by

$$
\operatorname{RMSE}=\left(\frac{1}{J} \sum_{j=1}^{J}\left(\widehat{\theta}_{j}-\theta_{j}\right)^{2}\right)^{1 / 2},
$$

where $J$ is the times of independent Monte Carlo trials, $\widehat{\theta}_{j}$ stands for parameter estimation, and $\theta_{j}$ is the true DOA or velocity for the $j$ th trial.

Following [1], we compute CRLB $\mathrm{J}^{-1}$ for the unknown parameter $\underline{\Theta}=[\mathrm{DOA}, \mathrm{DFS}]^{T}$. The CRLB is a lower bound for the covariance matrix of any unbiased estimator $\underline{\widehat{\Theta}}$. We use 


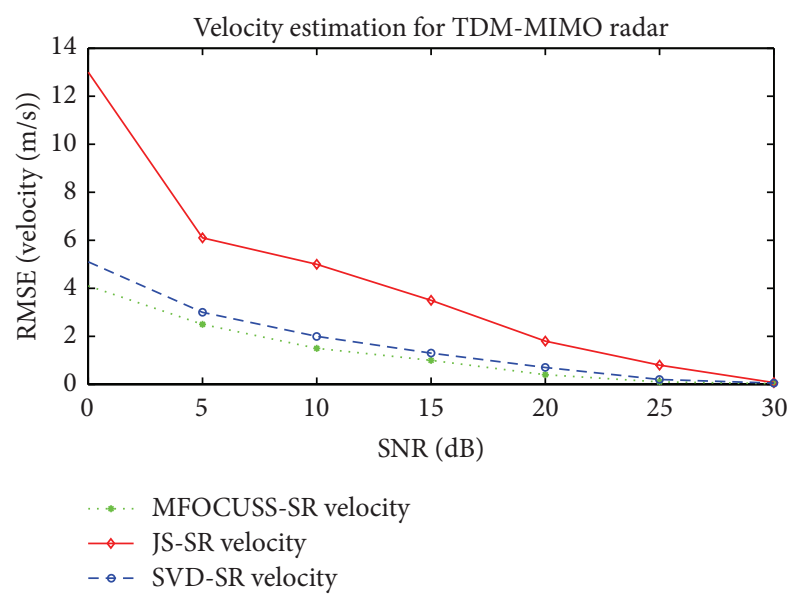

FIGURE 6: Velocity estimation for TDM-MIMO radar.

CRLB formula for DOA estimation $\left(\mathrm{CRB}_{\mathrm{DOA}}\right)$ according to paper [1]. The following formulas are the Cramer-Rao bounds of $\underline{\widehat{\Theta}}$ :

$$
\begin{aligned}
\mathbf{J}^{-1} & \frac{1}{2 L N_{\mathrm{RX}}} \frac{\sigma^{2}}{\sigma_{s}^{2}} \\
& \cdot 1 \times\left(\left(\operatorname{Var}^{S}\left(\underline{d}^{\mathrm{RX}}\right)+\operatorname{Var}^{S}\left(\underline{d}^{\text {Pulse }}\right)\right) \operatorname{Var}^{S}(\underline{t})\right. \\
& \left.-\left(\operatorname{Cov} S\left(\underline{d}^{\text {Pulse }}, \underline{t}\right)\right)^{2}\right)^{-1} \\
& \cdot\left[\begin{array}{c}
\operatorname{Var}^{S}(\underline{t}) \\
-\operatorname{Cov}^{S}\left(\underline{d}^{\text {Pulse }}, \underline{t}\right) \operatorname{Var}^{S}\left(\underline{d}^{\mathrm{RX}}\right)+\underline{\operatorname{Var}}^{S}\left(\underline{d}^{\text {Pulse }}\right)
\end{array}\right],
\end{aligned}
$$

$$
\begin{aligned}
\mathrm{CRB}_{\mathrm{DOA}} & \\
= & J_{11}^{-1} \\
= & \frac{1}{2 L N_{\mathrm{RX}}} \frac{\sigma^{2}}{\sigma_{s}^{2}} \cdot \operatorname{Var}^{S}(\underline{t}) \\
& \times\left(\left(\operatorname{Var}^{S}\left(\underline{d}^{\mathrm{RX}}\right)+\operatorname{Var}^{S}\left(\underline{d}^{\mathrm{Pulse}}\right)\right) \operatorname{Var}^{S}(\underline{t})\right. \\
& \left.\quad-\left(\operatorname{Cov}^{S}\left(\underline{d}^{\text {Pulse }}, \underline{t}\right)\right)^{2}\right)^{-1} .
\end{aligned}
$$

Here $\operatorname{Var}^{S}(\bullet)$ stands for sample variance and $\operatorname{Cov}^{S}(\bullet, *)$ stands for sample covariance. $\sigma_{s}^{2} / \sigma^{2}$ denotes the overall SNR. If an optimal TDM scheme is designed [1], $\mathrm{CRB}_{\mathrm{DOA}}$ will be minimized and $\left|\operatorname{Cov}{ }^{S}\left(\underline{d}^{\text {Pulse }}, \underline{t}\right)\right|$ will also be minimized to zero.

From the simulation results, we know that the error of angle measurements can reach below $0.2^{\circ}$ when SNR > $5 \mathrm{~dB}$ is satisfied for all three algorithms. But the error of velocity is not ideal for the JS-SR method under the same conditions. Only when SNR $>20 \mathrm{~dB}$ is satisfied, the error

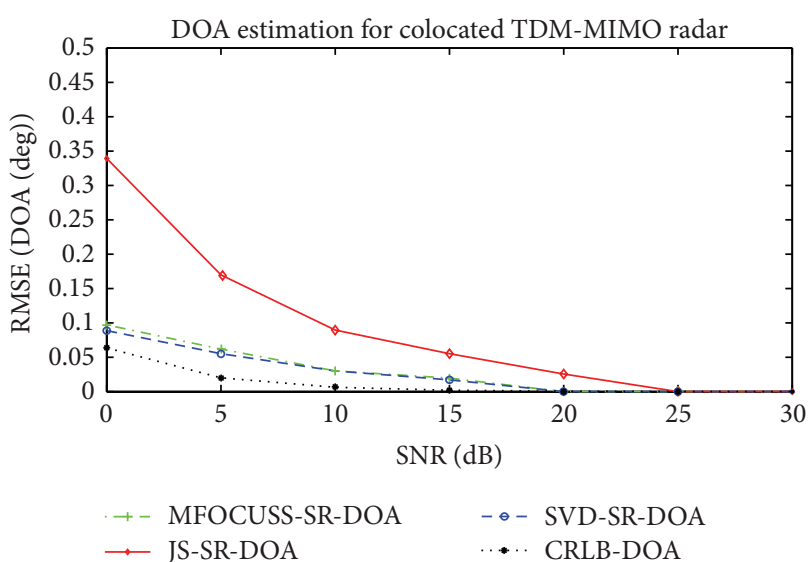

FIgURE 7: DOA estimation for TDM-MIMO radar.

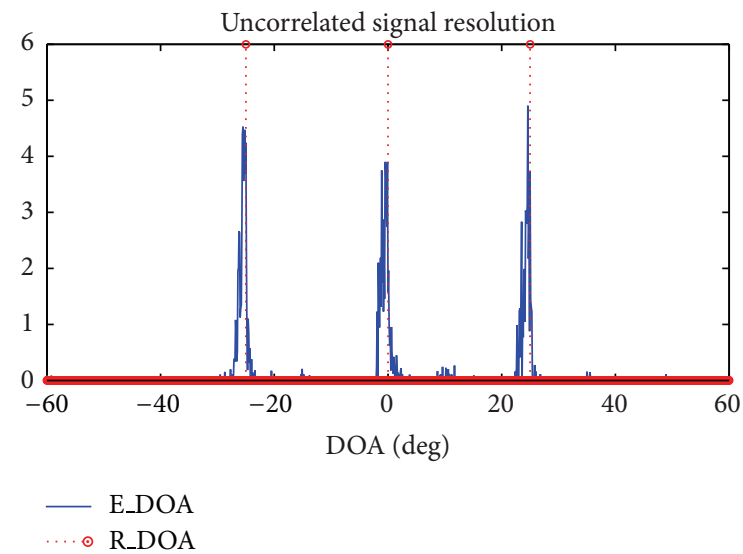

FIGURE 8: Uncorrelated signal resolution simulation based JS-SR.

of velocity will reach $v=2 \mathrm{~m} / \mathrm{s}$ below. But the SVDRS and MFOCUSS-RS algorithms can achieve significant performance improvement. DOA estimation results of these two algorithms are closer to the corresponding Cramer-Rao lower bound (CRLB) compared to JS-RS algorithm. So both MFOCUSS-RS and SVD-SR are superior to JS-SR algorithm.

5.2. Simulation Experiments of Multisignals Resolution. Here, we have utilized these three algorithms to test coherent and uncorrelated signals resolution. Experiments have the same input conditions that are $\mathrm{SNR}=20 \mathrm{~dB}$ and the true DOA of three simultaneous incident signals is $\left[\begin{array}{ccc}-25^{\circ} & 0^{\circ} & 25^{\circ}\end{array}\right]$. Correlated coefficient is defined by

$$
\rho_{i k}=\frac{E\left[s_{i}(t) s_{k}^{H}(t)\right]}{\sqrt{E\left[\left|s_{i}(t)\right|^{2}\right] E\left[\left|s_{k}(t)\right|^{2}\right]}} .
$$

Here $E[\bullet]$ denotes statistical expectation operator. $i, k$ denote two input signals. If $\rho_{i k}$ which lies in $[0,1]$ is equal to 1 , two input signals will be called as coherent signals.

The signal resolution simulation results are presented in Figure 8 to Figure 13. In Figures 8, 9, 10, 11, 12, and 13, E_DOA stands for estimation DOA and R_DOA stands for true DOA. 


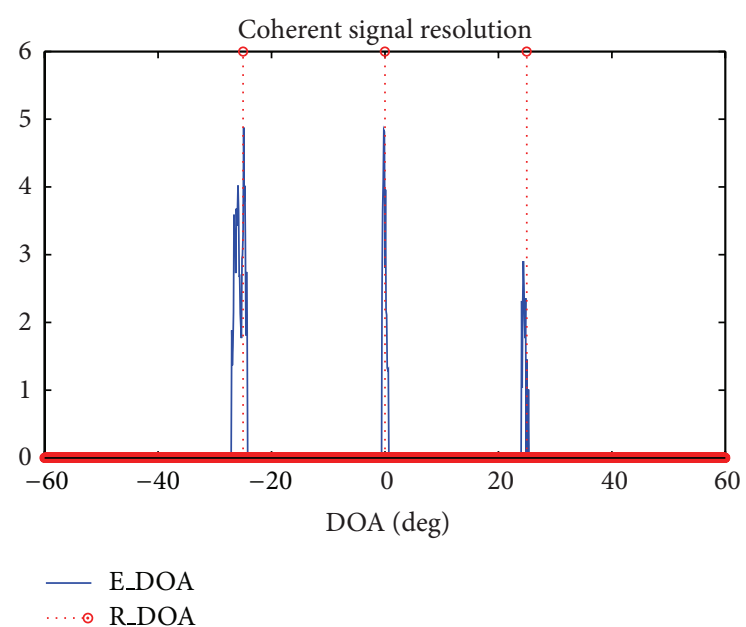

FIGURE 9: Coherent signal resolution simulation based JS-SR.

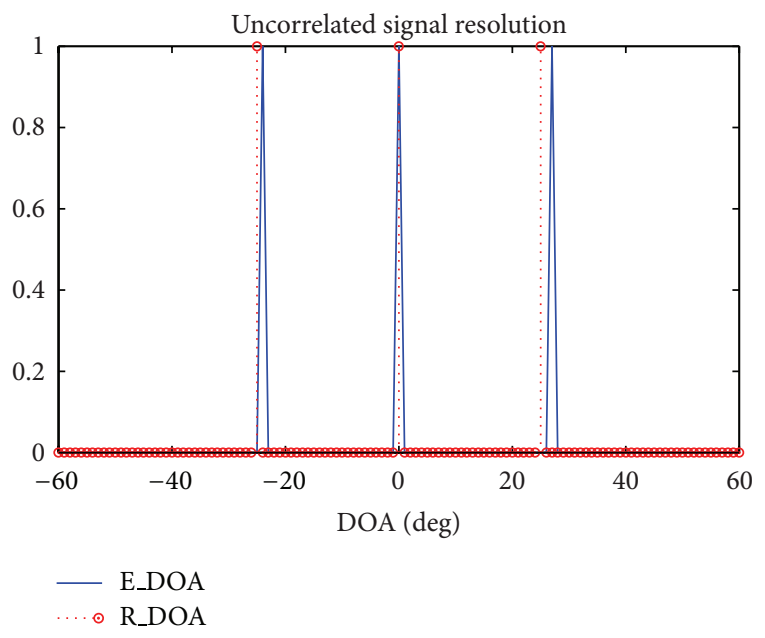

FIgURE 10: Uncorrelated signal resolution simulation based SVDSR.

All three algorithms can process uncorrelated signals. But there are some spurious peaks for resolution results of JS-SR approach and the magnitude of spurious peaks may be larger than the magnitude of true input signal in some cases like Figure 8. So it is very complex to seek the different peak values of true DOAs. The improved M-FOCUSS algorithm can work well. The magnitude of spurious peaks is smaller than the magnitude of true DOAs like in Figure 12. With the increase of SNR, the magnitude of spurious peaks can also become smaller and smaller. In the situation of coherent signals impinging, all three algorithms have an ability to complete multisource resolution. But the problem of spurious peaks still exists for JS-SR algorithm. Accuracy of DOA estimation for SVD-SR algorithm has deteriorated since the rank of matrix $\Lambda$ is reduced and then we have to select one vector corresponding to the largest singular value to calculate the sparse solution.

Above all, the improved M-FOCUSS algorithm can work more robust than SVD-SR and JS-SR algorithms. Multisource

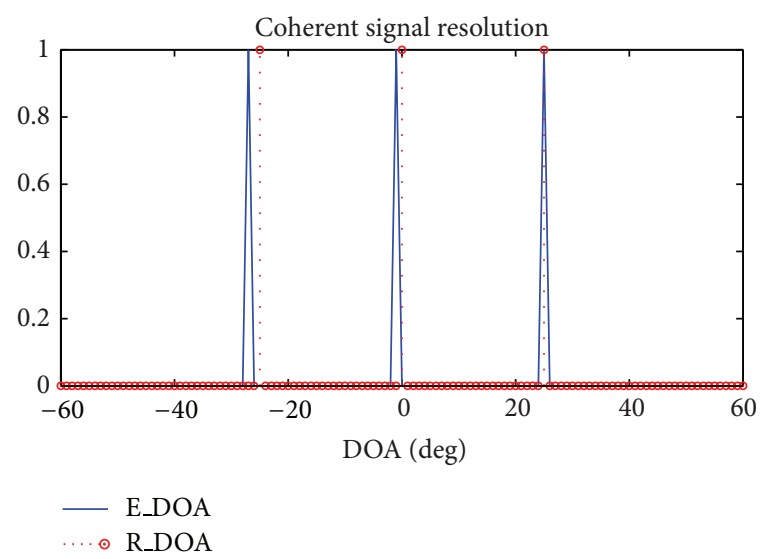

FIGURE 11: Coherent signal resolution simulation based SVD-SR.

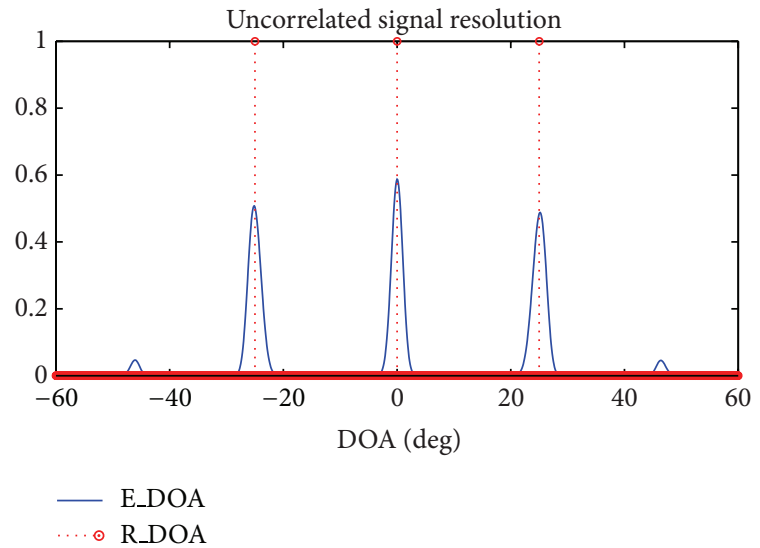

FIGURE 12: Uncorrelated signal resolution simulation based MFOCUSS-SR.

resolution is successful and DFS and DOA estimation values are very precise regardless of correlated or uncorrelated input signals when the improved M-FOCUSS algorithm is utilized to deal with TDM-MIMO model.

\section{Conclusions}

In this paper, we propose joint DFS and DOA estimation algorithm for colocated TDM-MIMO radar based signal sparse representations. Facing the problem of time varying steering vector, we establish input signal mode and depict the algorithms of sparse representations and signal reconstruction in detail. Three algorithms including joint sparse recovery spectrum, SVD decomposition with OMP algorithm, and M-FOCUSS are discussed to deal with MMV problem. The joint sparse recovery spectrum and M-FOCUSS methods are combined to improve M-FOCUSS performance in the case of MMV problem. Finally, the simulation results of multisource resolution and accuracy of estimations and some discussions are demonstrated. Using sparse representations based on the proposed angle-frequency space, the coherent signals can be distinguished and the Doppler frequency shift and DOA can be estimated jointly. Particularly, the joint estimation 


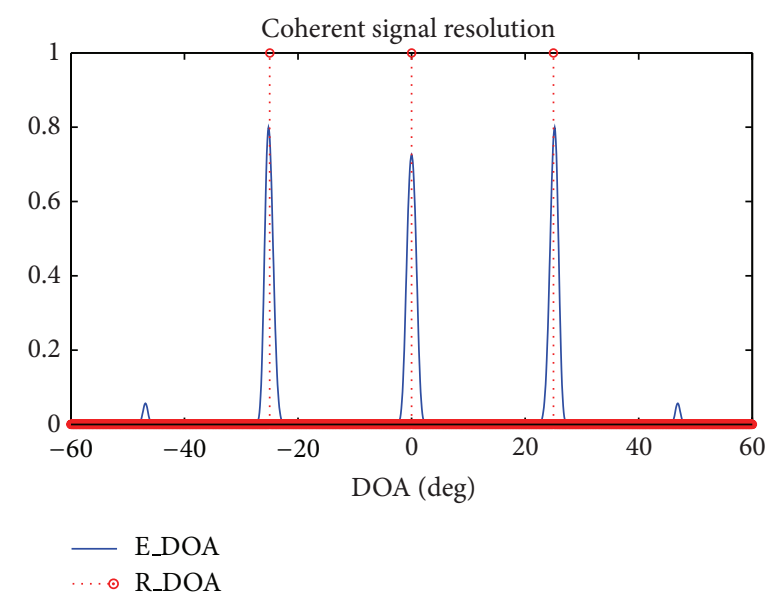

FIGURE 13: Coherent signal resolution simulation based MFOCUSSSR.

algorithm based on sparse representations using improved M-FOCUSS reconstruction can deal with MMV problem and achieve significant performance improvement of DOA and DFS estimation accuracy. So the proposed algorithm and model are effective and can be further applied for practical application.

\section{Conflict of Interests}

The authors declare that there is no conflict of interests regarding the publication of this paper.

\section{Acknowledgments}

This research paper is sponsored by China Scholarship Fund (CSC), in part supported by the Fundamental Research Funds for the Central Universities (HEUCF1408) and by the National Science Foundation of China under Grant no. 61201410 .

\section{References}

[1] K. Rambach and B. Yang, "Colocated MIMO radar: cramerRao bound and optimal time division multiplexing for DOA estimation of moving targets," in Proceedings of the 38th IEEE International Conference on Acoustics, Speech, and Signal Processing (ICASSP '13), pp. 4006-4010, Vancouver, Canada, May 2013.

[2] S. Lutz and T. Walter, "Lens based 77 GHz TDM MIMO radar sensor for angular estimation in multitarget environments," in Proceedings of the 10th European Radar Conference (EuRAD '13), pp. 212-215, October 2013.

[3] M. Rossi, A. M. Haimovich, and Y. C. Eldar, "Spatial compressive sensing for MIMO radar," IEEE Transactions on Signal Processing, vol. 62, no. 2, pp. 419-430, 2014.

[4] Y. Yu, A. P. Petropulu, and H. V. Poor, "MIMO radar using compressive sampling," IEEE Journal on Selected Topics in Signal Processing, vol. 4, no. 1, pp. 146-163, 2010.

[5] J. Liu, C. Han, and Y. Hu, "A novel coherent column replacement method in compressed sensing for DOA estimation," in
Proceedings of the 32nd Chinese Control Conference (CCC '13), pp. 4832-4837, IEEE, July 2013.

[6] J. Yin and T. Chen, "Direction-of-arrival estimation using a sparse representation of array covariance vectors," IEEE Transactions on Signal Processing, vol. 59, no. 9, pp. 4489-4493, 2011.

[7] J.-F. Gu, W.-P. Zhu, and M. N. S. Swamy, "Compressed sensing for DOA estimation with fewer receivers than sensors," in Proceedings of the IEEE International Symposium of Circuits and Systems (ISCAS '11), pp. 1752-1755, IEEE, May 2011.

[8] M.-S. Lee, V. Katkovnik, and Y.-H. Kim, "System modeling and signal processing for a switch antenna array radar," IEEE Transactions on Signal Processing, vol. 52, no. 6, pp. 1513-1523, 2004.

[9] S. Yin, G. Wang, and X. Yang, "Robust PLS approach for KPIrelated prediction and diagnosis against outliers and missing data," International Journal of Systems Science, vol. 45, no. 7, pp. 1375-1382, 2014.

[10] A. Zwanetski and H. Rohling, "Continuous wave MIMO radar based on time division multiplexing," in Proceedings of the 13th International Radar Symposium (IRS '12), pp. 119-121, IEEE, May 2012.

[11] L. Liu, Y. Jiang, L. Wan, and Z. Tian, "Beamforming of joint polarization-space matched filtering for conformal array," The Scientific World Journal, vol. 2013, Article ID 589675, 10 pages, 2013.

[12] L.-T. Wan, L.-T. Liu, W.-J. Si, and Z.-X. Tian, "Joint estimation of 2D-DOA and frequency based on space-time matrix and conformal array," The Scientific World Journal, vol. 2013, Article ID 463828, 10 pages, 2013.

[13] H. Feng, L. Liu, and B. Wen, "2D-DOA estimation for cylindrical array with mutual coupling," Mathematical Problems in Engineering, vol. 2014, Article ID 716978, 8 pages, 2014.

[14] S. Yin, G. Wang, and H. R. Karimi, "Data-driven design of robust fault detection system for wind turbines," Mechatronics, vol. 24, no. 4, pp. 298-306, 2014.

[15] M. Jahn, R. Feger, C. Wagner, Z. Tong, and A. Stelzer, "A four-channel 94-GHz sige-based digital beamforming FMCW radar," IEEE Transactions on Microwave Theory and Techniques, vol. 60, no. 3, pp. 861-869, 2012.

[16] P. Schmalenberg, J. S. Lee, and K. Shiozaki, "A SiGe-based 16channel phased array radar system at W-Band for automotive applications," in Proceedings of the 10th European Radar Conference (EuRAD '13), pp. 299-302, October 2013.

[17] S. Yin, X. Yang, and H. R. Karimi, "Data-driven adaptive observer for fault diagnosis," Mathematical Problems in Engineering, vol. 2012, Article ID 832836, 21 pages, 2012.

[18] P. Wenig, M. Schoor, O. Gunther, B. Yang, and R. Weigel, "System design of a $77 \mathrm{GHz}$ automotive radar sensor with superresolution DOA estimation," in Proceedings of the IEEE International Symposium on Signals, Systems and Electronics (ISSSE '07), pp. 537-540, August 2007.

[19] K. Rambach and B. Yang, "Direction of Arrival estimation of two moving targets using a time division multiplexed colocated MIMO radar," in Proceedings of the IEEE Radar Conference, pp. 1118-1123, Cincinnati, Ohio, USA, May 2014.

[20] T. Pedersen, C. Pedersen, X. Yin et al., "Joint estimation of doppler frequency and directions in channel sounding using switched Tx and Rx arrays," in Proceedings of the IEEE Global Telecommunications Conference (GLOBECOM '04), vol. 4, pp. 2354-2360, December 2004. 
[21] B. D. Rao, K. Engan, and S. Cotter, "Diversity measure minimization based method for computing sparse solutions to linear inverse problems with multiple measurement vectors," in Proceedings of the IEEE International Conference on Acoustics, Speech, and Signal Processing (ICASSP '04), vol. 2, pp. II369II372, IEEE, May 2004.

[22] Y. Wang, T. Fu, M. Gao, and S. Ding, "DOA estimation by exploiting spatial and Doppler sparsity," in Proceedings of the IET International Radar Conference, pp. 1-4, Xian, China, April 2013.

[23] B. Li, Y. Zou, and Y. Zhu, "Direction estimation under compressive sensing framework: a review and experimental results," in Proceedings of the International Conference on Information and Automation (ICIA '11), pp. 63-68, June 2011.

[24] Y. He, K. Hueske, E. Coersmeier, and J. Gdtze, "Efficient computation of joint direction-of-arrival and frequency estimation," in Proceedings of the 8th IEEE International Symposium on Signal Processing and Information Technology (ISSPIT '08), pp. 144149, IEEE, December 2008.

[25] Y. Wang, G. Leus, and A. Pandharipande, "Direction estimation using compressive sampling array processing," in Proceedings of the IEEE/SP 15th Workshop on Statistical Signal Processing (SSP '09), pp. 626-629, September 2009.

[26] A. Gretsistas and D. M. Plumbley, "An alternating descent algorithm for the off-grid DOA estimation problem with sparsity constraints," in Proceedings of the 20th European Signal Processing Conference (EUSIPCO '12), pp. 874-878, August 2012.

[27] D. Malioutov, M. Cetin, and A. S. Willsky, "A sparse signal reconstruction perspective for source localization with sensor arrays," IEEE Transactions on Signal Processing, vol. 53, no. 8, pp. 3010-3022, 2005.

[28] S. Yin, X. Li, H. Gao, and O. Kaynak, "Data-based techniques focused on modern industry: an overview," IEEE Transactions on Industrial Electronics, 2014.

[29] S. Yin, S. X. Ding, X. Xie, and H. Luo, "A review on basic datadriven approaches for industrial process monitoring," IEEE Transactions on Industrial Electronics, vol. 61, no. 11, pp. 64186428, 2014.

[30] I. F. Gorodnitsky and B. D. Rao, "Sparse signal reconstruction from limited data using FOCUSS: a re-weighted minimum norm algorithm," IEEE Transactions on Signal Processing, vol. 45, no. 3, pp. 600-616, 1997. 


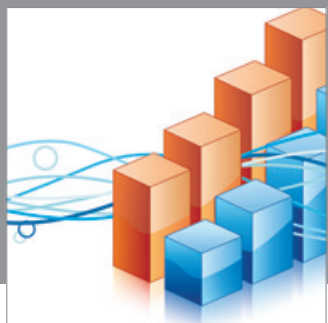

Advances in

Operations Research

mansans

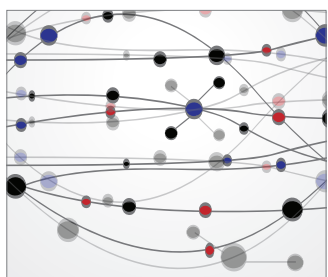

The Scientific World Journal
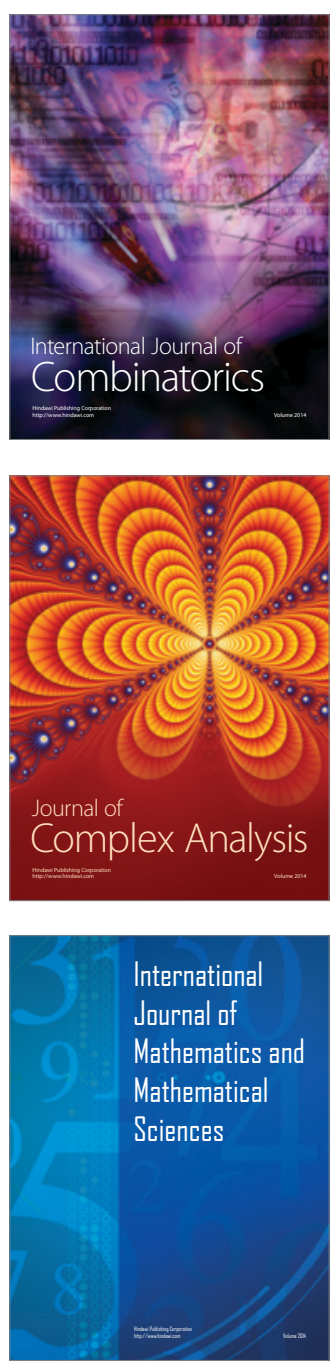
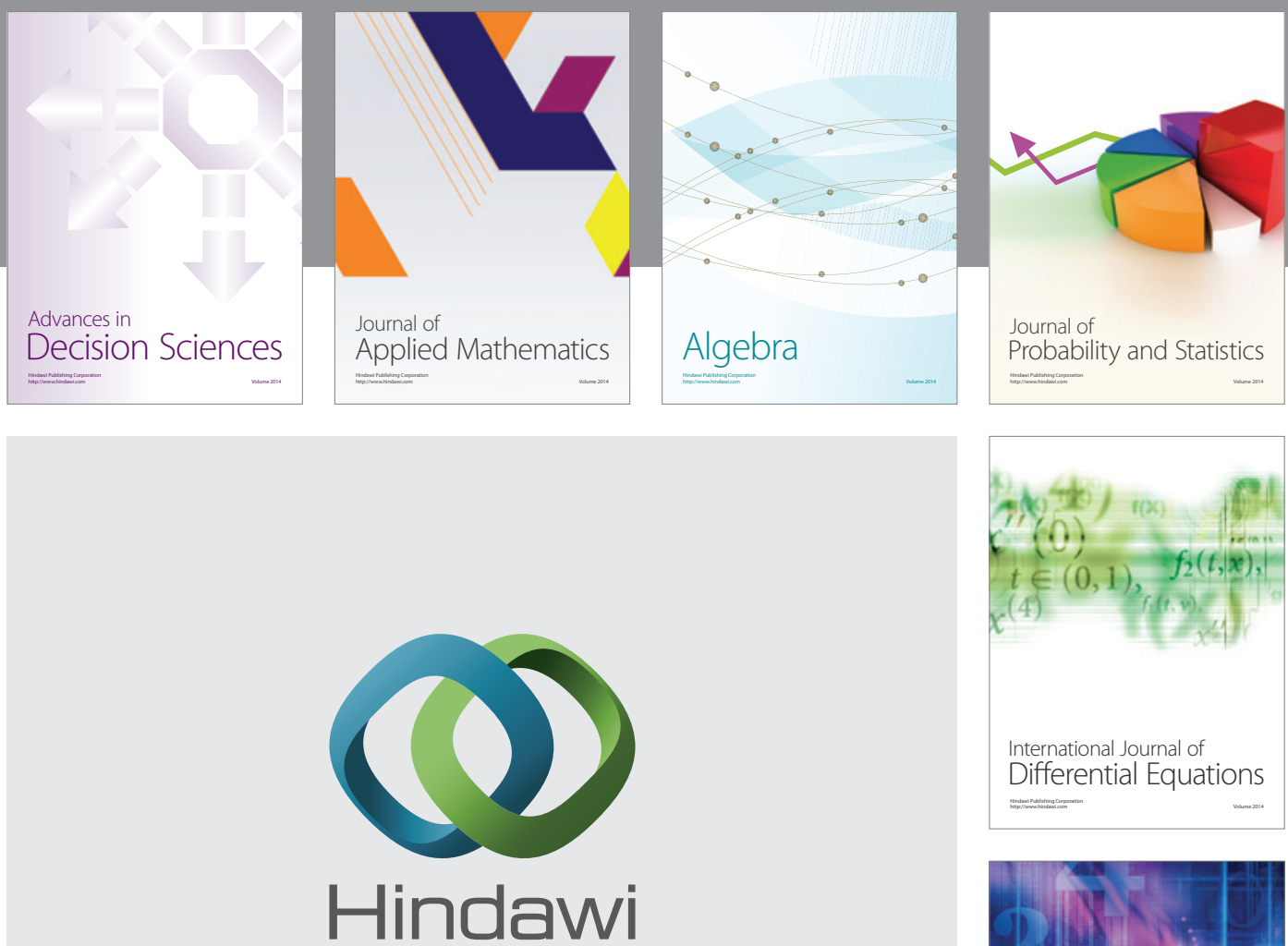

Submit your manuscripts at http://www.hindawi.com
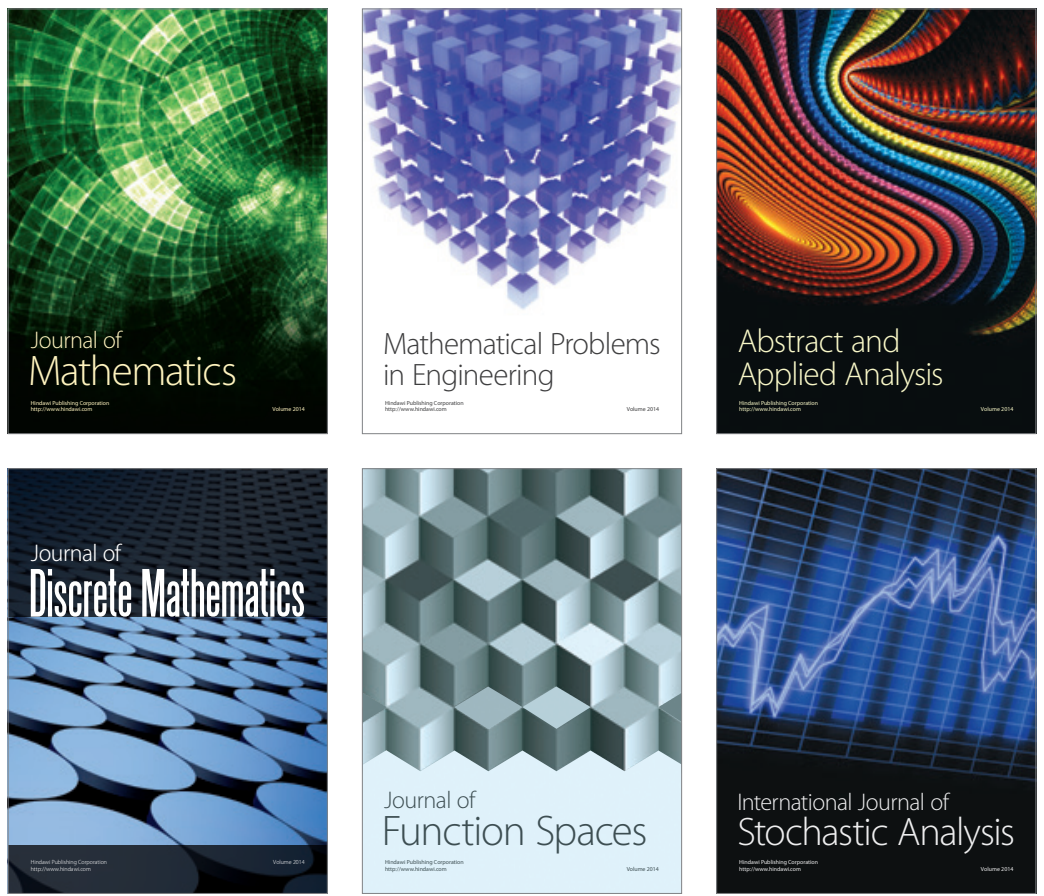

Journal of

Function Spaces

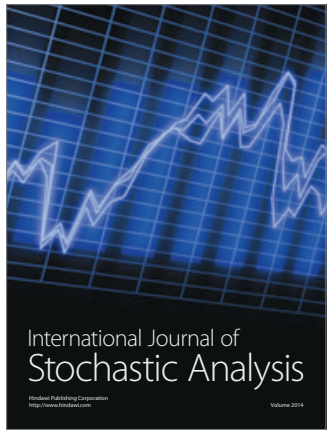

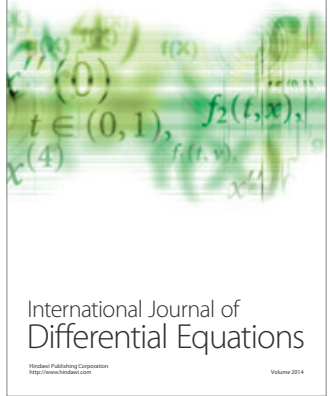
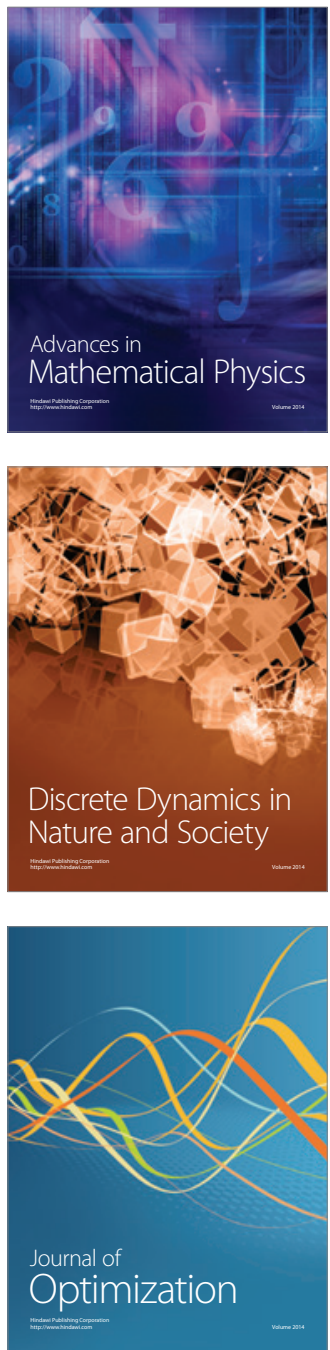